\title{
Length-type parameters of finite groups with almost unipotent automorphisms
}

\section{E. I. Khukhro, P. Shumyatsky}

1. Introduction. By Baer's theorem [2, Satz III.6.15], an element $g$ of a finite group $G$ belongs to the Fitting subgroup $F(G)$ if and only if it is a left-Engel element, that is, $[x, g, g, \ldots, g]=1$ for all $x \in G$, where $g$ is repeated sufficiently many times. (We use the notation for left-normed simple commutators $\left[a_{1}, a_{2}, a_{3}, \ldots, a_{r}\right]=\left[\ldots\left[\left[a_{1}, a_{2}\right], a_{3}\right], \ldots, a_{r}\right]$.)

Many results about groups can be equivalently stated in terms of their automorphisms, as every element acting by conjugation induces an inner automorphism. In particular, Baer's theorem is equivalent to saying that if an automorphism $\alpha$ of a finite group $G$ is unipotent in the sense that $[x, \alpha, \alpha, \ldots, \alpha]=1$ for all $x \in G$, where $\alpha$ is repeated sufficiently many times, then the commutator subgroup $[G, \alpha]=\langle[g, \alpha] \mid g \in G\rangle$ is nilpotent. Here the commutators are understood in the sense of the semidirect product $G\langle\alpha\rangle$, that is, $[g, \alpha]=g^{-1} g^{\alpha}$. In this and other relevant results on automorphisms it is important that the order of the automorphism is not supposed to be coprime to the order of the group. Moreover, the results of the present paper are proved by considering automorphisms, and therefore it is natural to state them in these terms.

We generalize Baer's theorem using the subgroups $E_{G, n}(\alpha)=\langle[x, \underbrace{\alpha, \ldots, \alpha}_{n}] \mid x \in G\rangle$, where $\alpha$ is an automorphism of a finite group $G$. A condition that a certain parameter of the subgroup $E_{G, n}(\alpha)$ is small, in one sense or another, can be regarded as assuming that the automorphism $\alpha$ is "almost unipotent". The corresponding parameters considered in the present paper are the Fitting height in the case of soluble groups, and the nonsoluble length and generalized Fitting height in the case of nonsoluble groups.

Note that $E_{G, n}(\alpha)$ is not a subgroup of the type $[\ldots[[G, \alpha], \alpha], \ldots, \alpha]$ formed by taking successive commutator subgroups, which is subnormal. But the $\operatorname{subgroup} E_{G, n}(\alpha)$ is not subnormal in general.

2. Bounding the Fitting height. The Fitting series starts with the Fitting subgroup $F_{1}(G)=F(G)$, the largest nilpotent normal subgroup, and then by induction $F_{k+1}(G)$ is 
the inverse image of $F\left(G / F_{k}(G)\right)$. The Fitting height of a finite soluble group $G$ is the least positive integer $l$ such that $F_{l}(G)=G$.

Theorem 1. Let $\alpha$ be an automorphism of a finite soluble group $G$, and $n$ a positive integer. If the Fitting height of $E_{G, n}(\alpha)$ is equal to $k$, then the Fitting height of $[G, \alpha]$ is at most $k+1$.

The proof of Theorem 1 reduces to the following proposition.

Proposition 1. Let $\alpha$ be an automorphism of a finite soluble group $G$ such that $G=$ $[G, \alpha]$. Then $E_{G, n}(\alpha)=G$ for any $n$.

This proposition is proved by induction on the order of $G$.

3. Bounding the nonsoluble length. The nonsoluble length $\lambda(G)$ of a finite group $G$ is defined as the minimum number of nonsoluble factors in a normal series each of whose factors either is soluble or is a direct product of nonabelian simple groups. Bounds for the nonsoluble length of a finite group $G$ greatly facilitate using the classification of finite simple groups, and these bounds themselves are often obtained by using the classification. One of most notable applications of such bounds were in the reduction of the Restricted Burnside Problem to soluble and nilpotent groups in the Hall and Higman paper [1]. Such bounds also find applications in the study of profinite groups, for example, in Wilson's reduction [5] of the problem of local finiteness of periodic profinite groups to pro- $p$ groups, and in our recent paper [3] on similar problems. (Both the Restricted Burnside Problem and the problem of local finiteness of periodic profinite groups were solved by Zelmanov $[6,7,8]$.

Theorem 2. Let $m$ and $n$ be positive integers, and let $\alpha$ be an automorphism of a finite group $G$ whose order $|\alpha|$ is equal to the product of $m$ primes counting multiplicities. Let the nonsoluble length of $E_{G, n}(\alpha)$ be equal to $k$. Then the nonsoluble length of $[G, \alpha]$ is bounded in terms of $k$ and $m$.

The proof of Theorem 2 depends on the classification of finite simple group in so far as the validity of the Schreier conjecture on solubility of the group of outer automorphisms of a finite simple group.

First we analyse automorphisms of direct products of nonabelian finite simple groups. One of the typical lemmas is as follows. 
Lemma 1. Let $S=S_{1} \times \cdots \times S_{r}$ be a direct product of $r$ isomorphic finite non-abelian simple groups and let $\varphi$ be the natural automorphism of $S$ of order $r$ that regularly permutes the $S_{i}$. Let $n$ be a positive integer. Then $E_{S, n}(\varphi)=S$.

An important role in the proof of Theorem 2 is played by results on permutational actions of certain finite groups $G$ concerning the existence of exact (regular) orbits of an element $g \in G$. The proof of Theorem 2 requires rather difficult arguments using induction on a complex parameter. We refer the reader to the detailed exposition in [4].

4. Bounding the generalized Fitting height. The generalized Fitting subgroup $F^{*}(G)$ of a finite group $G$ is the product of the Fitting subgroup $F(G)$ and all subnormal quasisimple subgroups (here a group is quasisimple if it is equal to its derived subgroup and its quotient by the centre is a non-abelian simple group). Then the generalized Fitting series of $G$ is defined starting from $F_{1}^{*}(G)=F^{*}(G)$, and then by induction $F_{i+1}^{*}(G)$ is the inverse image of $F^{*}\left(G / F_{i}^{*}(G)\right)$. The least number $h$ such that $F_{h}^{*}(G)=G$ is defined to be the generalized Fitting height $h^{*}(G)$ of $G$.

Theorem 3. Let $m$ and $n$ be positive integers, and let $\alpha$ be an automorphism of a finite group $G$ whose order $|\alpha|$ is equal to the product of $m$ primes counting multiplicities. Let the generalized Fitting height of $E_{G, n}(\alpha)$ be equal to $k$. Then the generalized Fitting height of $[G, \alpha]$ is bounded in terms of $k$ and $m$.

This theorem follows from Theorem 1 on soluble groups and Theorem 2 on the nonsoluble length.

5. Final remarks and conjectures. In Theorems 2 and 3, the nonsoluble length and generalized Fitting height are given bounds depending both on the parameters of $E_{G, n}(\alpha)$ and on the number of prime factors in the order of $\alpha$. We conjecture that stronger results may hold, not depending on the order of $\alpha$.

Conjecture 1. Let $\alpha$ be an automorphism of a finite group $G$, and $n$ a positive integer. If the nonsoluble length of $E_{G, n}(\alpha)$ is equal to $k$, then the nonsoluble length of $[G, \alpha]$ does not exceed $k+1$.

Conjecture 2. Let $\alpha$ be an automorphism of a finite group $G$, and $n$ a positive integer. If the generalized Fitting height of $E_{G, n}(\alpha)$ is equal to $k$, then the generalized Fitting height of $[G, \alpha]$ does not exceed $k+1$. 
We prove that Conjectures 1 and 2 can be reduced to the following conjecture.

Conjecture 3. Let $S=S_{1} \times \cdots \times S_{r}$ be a direct product of nonabelian finite simple groups, and $\varphi$ an automorphism of $S$ transitively permuting the factors. Then $E_{S, n}(\varphi)=S$.

So far we have shown in Lemma 1 that Conjecture 3 holds in the special case where the order of $\varphi$ is equal to the number of factors $r$. However, in general Conjecture 3 seems rather difficult and remains unproved. The first step would be to consider the case of $\varphi$ of prime order, when of course the open question is about a single nonabelian finite simple group and its automorphism of prime order. A significant headway in this direction was recently made by Robert Guralnick (private communication).

Theorem 4. Conjectures 1 and 2 are true if Conjecture 3 is true.

Acknowledgments. The first author was supported by the Russian Science Foundation, project no. 14-21-00065, and the second by CNPq, Brazil.

The authors thank Professor Robert M. Guralnick for useful discussions.

\section{References}

[1] P. Hall and G. Higman, Proc. London Math. Soc. (3), 6 (1956), 1-42.

[2] B. Huppert, Endliche Gruppen. I, Springer, Berlin, 1967.

[3] E. I. Khukhro and P. Shumyatsky, J. Austral. Math. Soc. 97 (2014), 343-364.

[4] E. I. Khukhro and P. Shumyatsky, Preprint, 2015; arxiv.org/abs/1506.00233.

[5] J. Wilson, Monatsh. Math., 96 (1983), 57-66.

[6] E. I. Zelmanov, Izv. Akad. Nauk SSSR Ser. Mat., 54, 42-59; English transl., Math. USSR Izvestiya, 36 (1991), 41-60.

[7] E. I. Zelmanov, Mat. Sbornik, 182, 568-592; English transl., Math. USSR Sbornik, 72 (1992), 543-565.

[8] E. I. Zelmanov, Israel J. Math. 77 (1992), 83-95. 


\section{AUTOREFERAT}

\section{Length-type parameters of finite groups}

\section{with almost unipotent automorphisms}

\section{E. I. Khukhro, P. Shumyatsky}

Let $\alpha$ be an automorphism of a finite group $G$. For a positive integer $n$, let $E_{G, n}(\alpha)$ be the subgroup generated by all commutators $[\ldots[[x, \alpha], \alpha], \ldots, \alpha]$ in the semidirect product $G\langle\alpha\rangle$ over $x \in G$, where $\alpha$ is repeated $n$ times. By Baer's theorem, if $E_{G, n}(\alpha)=1$, then the commutator subgroup $[G, \alpha]$ is nilpotent. We generalize this theorem in terms of certain length parameters of $E_{G, n}(\alpha)$. For soluble $G$ we prove that if, for some $n$, the Fitting height of $E_{G, n}(\alpha)$ is equal to $k$, then the Fitting height of $[G, \alpha]$ is at most $k+1$. For nonsoluble $G$ the results are in terms of the nonsoluble length and generalized Fitting height. The generalized Fitting height $h^{*}(H)$ of a finite group $H$ is the least number $h$ such that $F_{h}^{*}(H)=H$, where $F_{0}^{*}(H)=1$, and $F_{i+1}^{*}(H)$ is the inverse image of the generalized Fitting subgroup $F^{*}\left(H / F_{i}^{*}(H)\right)$. Let $m$ be the number of prime factors of the order $|\alpha|$ counting multiplicities. It is proved that if, for some $n$, the generalized Fitting height of $E_{G, n}(\alpha)$ is equal to $k$, then the generalized Fitting height of $[G, \alpha]$ is bounded in terms of $k$ and $m$. The nonsoluble length $\lambda(H)$ of a finite group $H$ is defined as the minimum number of nonsoluble factors in a normal series each of whose factors either is soluble or is a direct product of nonabelian simple groups. It is proved that if $\lambda\left(E_{G, n}(\alpha)\right)=k$, then the nonsoluble length of $[G, \alpha]$ is bounded in terms of $k$ and $m$. We also state conjectures of stronger results independent of $m$ and show that these conjectures reduce to a certain question about automorphisms of direct products of finite simple groups. 